\title{
Interactions of Ibuprofen with Influenza Infection and Hyperammonemia in an Animal Model of Reye's Syndrome
}

\author{
ARINDAM MUKHOPADHYAY, ASHOK P. SARNAIK, AND DEVENDRA R. DESHMUKH \\ Department of Pediatrics, Children's Hospital of Michigan and Wayne State University, School of Medicine, \\ Detroit, Michigan 48201
}

\begin{abstract}
We have previously reported that a single meal of an arginine-free diet rapidly induces hyperammonemia in young ferrets and that aspirin administration in conjunction with influenza $B$ infection and arginine-free diet results in clinical and biochemical alterations consistent with Reye's syndrome. The objective of the present study was to test whether ibuprofen administration, either alone or in combination with influenza infection and arginine-free diet, produces a similar effect. Two-mo-old ferrets were inoculated intranasally with influenza $B$ virus, treated with therapeutic doses of ibuprofen, and fed a single meal of an arginine-free diet. Arginine-free diet caused a significant increase in plasma ammonia and a small increase in plasma aspartate aminotransferase activity. All ferrets fed an arginine-free diet recovered within 6 to $7 \mathrm{~h}$ after ingesting the diet. Ibuprofen treatment, either solely or in combination with influenza infection, did not produce significant change in the plasma levels of aspartate or ornithine aminotransferase activities. A combination of ibuprofen treatment, influenza infection, and arginine-free diet caused a significant increase in the mortality and plasma ammonia levels. Plasma aspartate aminotransferase and ornithine carbamyl transferase activities were elevated, and liver ornithine carbamyl transferase activity was decreased. However, other mitochondrial enzymes such as ornithine aminotransferase were not altered, whereas the activity of cytoplasmic enzymes such as arginase were decreased. These results suggest that ibuprofen administration resulted in generalized hepatopathy rather than specific mitochondrial injury and Reye's syndrome-like changes associated with aspirin in our previous model. (Pediatr Res 31: 258-260, 1992)
\end{abstract}

\section{Abbreviations}

ACD, arginine-containing diet

AFD, arginine-free diet

Reye's syndrome, an encephalopathy with fatty degeneration of the viscera, is a rare but often fatal childhood disorder (1). Reye's syndrome is initiated by a prodromal viral illness, followed by vomiting, disorientation, seizures, and loss of consciousness. Clinical features of Reye's syndrome include stereotypic encephalopathy, hyperammonemia, and elevated serum aspartate and alanine aminotransferase activities with no change

Received August 14, 1991; accepted October 10, 1991.

Correspondence: Devendra R. Deshmukh, Department of Pediatrics, Critical Care Medicine Division, Children's Hospital of Michigan, 3901 Beaubien Blvd., Detroit, MI 48201 in serum bilirubin. In addition, several biochemical, clinical, and histopathologic abnormalities are associated with disturbances in the metabolism of amino acids, carbohydrates, and lipids (2-6).

Aspirin has been strongly implicated as one of the factors in the development of Reye's syndrome (7-10). Aspirin products carry a label with a warning to consumers of an association with Reye's syndrome. Ibuprofen has been widely used in children as an analgesic and antipyretic. However, whether ibuprofen precipitates Reye's syndrome-like disorder has not been investigated. Our previous studies revealed a synergistic effect of influenza B infection, aspirin ingestion, and hyperammonemia induced by an AFD in young ferrets (11). The objective of the present study was to test whether ibuprofen, either alone or in combination with influenza infection and hyperammonemia, produces the biochemical and clinical abnormalities characteristic of Reye's syndrome in the ferret model.

\section{MATERIALS AND METHODS}

Chemicals. Ibuprofen, amino acids, and other chemicals were purchased from Sigma Chemical Co. (St. Louis, MO). Vitamin mixture and starch were from ICN Pharmaceuticals (Cleveland, $\mathrm{OH})$.

Animals. Two-mo-old male, sable-coated ferrets, vaccinated for canine distemper, were purchased from Marshall Research Farms, North Rose, NY. They were housed in groups of three in cages with grid flooring in an isolation room with controlled light and temperature. The study was approved by the Institutional Animal Care and Use Committee.

Diets. Water and stock diet (Ferret Chow, Ralston Purina Co., St. Louis, MO) were provided ad libitum unless otherwise indicated. ACD was prepared as described by Morris and Rogers (12), except that corn oil was used instead of turkey fat. This diet consists of free amino acids, carbohydrates, commercial vitamins, and salt mixtures. An AFD was prepared by substituting alanine for an isonitrogenous amount of arginine.

Infection. Ferrets were infected with influenza $\mathrm{B}(\mathrm{B} / \mathrm{Bec} / 72)$ by inoculating $0.5 \mathrm{~mL}$ of virus into each nostril. The viral titer was $10^{6} 50 \%$ infective dose $/ \mathrm{mL}$ as measured with hen eggs. Racks of cages with infected ferrets were separated from those of noninfected animals.

Ibuprofen treatment. Ibuprofen was dissolved in water, neutralized with $1 \mathrm{M}$ sodium hydroxide, and delivered by feeding tube (30 mg/kg, twice daily).

Experimental design. Ferrets were divided into eight groups, with at least five animals in each group, designated as follows: 1) control, 2) influenza, 3) ibuprofen, 4) influenza plus ibuprofen, 5) AFD, 6) influenza plus AFD, 7) ibuprofen plus AFD, and 8 ) influenza, ibuprofen, and AFD. On the 1 st $\mathrm{d}$ of the experiment, animals in groups $2,4,6$, and 8 were infected with influenza B virus. Ibuprofen treatment of ferrets in groups 3,4 , 7 , and 8 was started in the evening of the 1 st $d$. On the $3 r d d$, 
food was removed from all the cages. On the 4th d, an ACD was given to ferrets in groups 1-4 and AFD was provided to the ferrets in groups $5-8$.

In the first set of experiments, the duration, the severity of sickness, and mortality were studied. Animals were divided into eight groups as described above and observed for $5 \mathrm{~d}$. The second set of experiments was carried out as follows: On the 4th d, the ferrets were given the specified diet and, $3 \mathrm{~h}$ later, $3 \mathrm{~mL}$ of blood were collected by cardiac puncture in heparinized tubes (Vacutainer; Becton Dickinson, Rutherford, NJ). Blood was centrifuged at $5000 \times g$ in a refrigerated centrifuge. Plasma was removed and stored at $-70^{\circ} \mathrm{C}$. All animals were killed $6-7 \mathrm{~h}$ after they were fed the diet, and their livers were removed and stored at $-70^{\circ} \mathrm{C}$.

Immediately after blood collection, plasma ammonia was determined (13). Aspartate aminotransferase (EC 2.6.1.1) was assayed at $30^{\circ} \mathrm{C}(14)$, and ornithine carbamyl transferase (EC 2.1.3.3) was assayed at $37^{\circ} \mathrm{C}(15)$. Plasma level of ibuprofen was estimated by HPLC (16). A $10 \%$ liver homogenate in distilled water (cytoplasmic enzymes) or in distilled water containing $1 \%$ Triton X-100 (mitochondrial enzymes) was prepared. Liver arginase (17) (EC 3.5.3.1), ornithine-oxo-acid aminotransferase (18) (EC 2.6.1.13), and ornithine carbamyl transferase (15) activities were determined at $37^{\circ} \mathrm{C}$. Liver protein concentrations were measured by the protein dye-binding assay of Bradford (19), using BSA as a standard.

Statistical analysis. Data were analyzed using a one-way analysis of variance followed by the least significance difference test. A $p$ value less than 0.05 was considered statistically significant.

\section{RESULTS}

Preliminary results indicated that the amount of food intake between ACD and AFD groups was similar (about 15-20 g of diet). Control ferrets (group 1) fed ACD did not exhibit obvious sickness during the 5-d observation period. Ferrets fed ACD with or without infection did not develop hyperammonemia during a prolonged observation period (4-5 d). Ibuprofen treatment of noninfected (group 3) or infected (group 4) ferrets fed ACD did not cause obvious sickness. On the 3 rd d after inoculation with influenza B virus (groups 2 and 6), animals developed fever (rectal temperature $103-105^{\circ} \mathrm{F}$ ). Infected ferrets treated with ibuprofen (groups 3, 4, and 8) did not develop fever.

Influenza infection, ibuprofen treatment, or both did not cause any fatality (groups 2-4). Ferrets fed AFD (group 5) became hyperactive $2 \mathrm{~h}$ after ingesting the diet. Within $30 \mathrm{~min}$ of the onset of hyperactivity, the typical signs of hyperammonemia such as irritability and seizures were observed, followed by prostration and coma. All ferrets in group 5 survived the AFD challenge. A combination of influenza and AFD did not cause any death. One out of five ferrets treated with ibuprofen plus AFD died. Ferrets infected with influenza (group 6) or treated with ibuprofen (group 7) before feeding AFD remained sick for 4-5 h, whereas ferrets with influenza plus ibuprofen plus AFD (group 8) remained sick for 12-14 h. The mortality was also maximal in group 8.

Treatment with influenza, ibuprofen, or both to ferrets fed ACD (groups 2-4) did not alter the plasma ammonia levels or ornithine carbamyl transferase activity. Plasma aspartate aminotransferase activity in infected or ibuprofen-treated ferrets was increased as compared with controls (Table 1). A significant increase in plasma ammonia and aspartate aminotransferase levels were noted in ferrets fed AFD (group 5). Treatment with influenza infection (group 6) or ibuprofen (group 7) followed by AFD caused further increase in the plasma aspartate aminotransferase and ornithine carbamyl transferase activities (Table 1). The concentration of plasma ibuprofen was not affected by the infection or by AFD treatment (Table 1).

A combination of influenza B infection, ibuprofen treatment, and AFD (group 8) caused the maximum increase in plasma ammonium levels. Aspartate aminotransferase and ornithine carbamyl transferase activities were also elevated. The effect of ibuprofen, influenza, and AFD on the hepatic urea cycle enzyme activities is shown in Table 2. Only the ferrets that were infected with influenza, treated with ibuprofen, and fed AFD (group 8) exhibited a significant decrease in the hepatic arginase and ornithine carbamyl transferase activities as compared with controls. Hepatic ornithine aminotransferase activity was not altered in any group.

\section{DISCUSSION}

The role of virus, drugs, and their interaction with other etiologic agents is difficult to study in patients with Reye's syndrome because the viral infection and the exposure to drugs occur in the prodromal period. We have developed an animal model of Reye's syndrome in young male ferrets by infecting them with influenza $B$ virus, treating them with therapeutic doses of aspirin, and feeding them a small amount of an AFD (11). Ferrets were selected as a model because, unlike most other laboratory animals, they are extremely susceptible to human influenza virus (20).

Previous experiments from our laboratory showed about $10 \%$ mortality in ferrets fed AFD (11). Treatment with influenza B virus and aspirin followed by AFD frequently results in death within a few hours after feeding the diet. Animals treated with ibuprofen, influenza, and AFD showed a more protracted and less severe illness as compared with the aspirin-treated group. Whether ibuprofen treatment impairs the detoxification of ammonia in this model remains to be investigated.

Some of the biochemical abnormalities in Reye's syndrome include hyperammonemia, increase in plasma aspartate aminotransferase and alanine aminotransferase, and normal serum bilirubin. Although ferrets treated with ibuprofen, influenza B virus, and AFD exhibited elevated plasma aspartate aminotransferase and ornithine carbamyl transferase, the increase in plasma aspartate aminotransferase and ornithine carbamyl transferase was not as severe as that seen with aspirin (11).

High dose of ibuprofen is used to treat juvenile rheumatoid

Table 1. Effects of influenza, ibuprofen, and AFD on plasma parameters and death rate in ferrets*

\begin{tabular}{|c|c|c|c|c|c|}
\hline Group & $\begin{array}{c}\text { Ammonia } \\
(\mu \mathrm{mol} / \mathrm{L})\end{array}$ & $\begin{array}{c}\mathrm{AST} \\
(\mathrm{IU} / \mathrm{L})\end{array}$ & $\begin{array}{c}\text { OCT } \\
(\mathrm{IU} / \mathrm{L})\end{array}$ & $\begin{array}{c}\text { Ibuprofen } \\
(\mu \mathrm{mol} / \mathrm{L})\end{array}$ & Mortality \\
\hline 1. Control & $138 \pm 8$ & $34 \pm 3.5$ & $9.8 \pm 1.3$ & & $0 / 5$ \\
\hline 2. $\operatorname{Inf}+\mathrm{ACD}$ & $102 \pm 10$ & $45 \pm 3.5 \dagger$ & $10.8 \pm 1.0$ & & $0 / 5$ \\
\hline 3. $\mathrm{IBF}+\mathrm{ACD}$ & $107 \pm 11$ & $53 \pm 19 \dagger$ & $7.4 \pm 0.9$ & $198 \pm 30$ & $0 / 5$ \\
\hline 4. $\operatorname{Inf}+\mathrm{IBF}+\mathrm{ACD}$ & $127 \pm 22$ & $42 \pm 4.3$ & $8.1 \pm 0.8$ & $210 \pm 30$ & $0 / 5$ \\
\hline 5. AFD & $2637 \pm 83 \dagger$ & $50 \pm 1.6 \dagger$ & $9.5 \pm 1.0$ & & $0 / 5$ \\
\hline 6. $\operatorname{Inf}+\mathrm{AFD}$ & $2096 \pm 198 \dagger$ & $64 \pm 5.0 \dagger$ & $25.0 \pm 2.0 \dagger$ & & $0 / 5$ \\
\hline 7. $\mathrm{IBF}+\mathrm{AFD}$ & $3092 \pm 107 \dagger$ & $54 \pm 3.7 \dagger$ & $21.3 \pm 2.9 \dagger$ & $210 \pm 14$ & $1 / 5$ \\
\hline 8. $\operatorname{Inf}+\mathrm{IBF}+\mathrm{AFD}$ & $3925 \pm 155 \dagger$ & $85 \pm 12 \dagger$ & $73 \pm 7 \dagger$ & $234 \pm 7$ & $4 / 7$ \\
\hline
\end{tabular}

$*$ Results are mean $\pm \operatorname{SEM}(n=5)$. AST, aspartate aminotransferase; OCT, ornithine carbamyl transferase.

$\dagger$ Statistically significant difference $(p<0.05)$ compared with controls. 
Table 2. Effects of influenza, ibuprofen, and AFD on urea cycle enzyme activities in ferret liver*

\begin{tabular}{lcccl}
\hline \multicolumn{1}{c}{ Group } & \multicolumn{2}{c}{$\begin{array}{c}\text { Arginase } \\
(\mathrm{nmol} / \mathrm{min} / \mathrm{mg} \text { protein })\end{array}$} & $\begin{array}{c}\text { OCT } \\
\text { (mg/g) }\end{array}$ \\
\hline 1. Control & $1193 \pm 44$ & $919 \pm 33$ & $9.8 \pm 0.5$ & $131 \pm 14$ \\
2. Inf + ACD & $907 \pm 92$ & $847 \pm 16$ & $11.2 \pm 0.9$ & $124 \pm 6$ \\
3. IBF + ACD & $1069 \pm 123$ & $955 \pm 50$ & $8.6 \pm 0.3$ & $157 \pm 10$ \\
4. Inf + IBF + ACD & $964 \pm 41$ & $888 \pm 43$ & $10.5 \pm 0.9$ & $152 \pm 6$ \\
5. AFD & $1209 \pm 50$ & $915 \pm 23$ & $11.5 \pm 1.2$ & $150 \pm 6$ \\
6. Inf + AFD & $1033 \pm 123$ & $820 \pm 27$ & $14.0 \pm 0.9$ & $143 \pm 6$ \\
7. IBF + AFD & $909 \pm 52$ & $757 \pm 66 \dagger$ & $10.0 \pm 0.7$ & $132 \pm 4$ \\
8. Inf + IBF + AFD & $759 \pm 70 \dagger$ & $559 \pm 24 \dagger$ & $9.5 \pm 0.4$ & $128 \pm 5$ \\
\hline
\end{tabular}

* Results are mean \pm SEM $(n=5)$. OCT, ornithine carbamyl transferase; OAT, ornithine aminotransferase.

$\dagger$ Statistically significant difference $(p<0.05)$ compared with controls.

arthritis and nonrheumatoid arthropathies (21). Ibuprofen taken orally is absorbed from the upper gastrointestinal tract and rapidly metabolized and excreted by the kidney. We selected the dosage of ibuprofen $(30 \mathrm{mg} / \mathrm{kg})$ to approximate maximum but nontoxic dose. Plasma ibuprofen levels were not affected by influenza infection or by the diet-induced hyperammonemia.

Biochemical and histopathologic studies provide evidence of acute mitochondrial insult in Reye's syndrome $(6,22)$. These reports suggest that the metabolic abnormalities seen in Reye's syndrome could be due to the mitochondrial dysfunction. In the liver of Reye's syndrome patients, all cytoplasmic enzyme activities measured, including arginase and argininosuccinate synthetase, were normal. Conversely, tissue activities of all of the mitochondrial enzyme activities including ornithine carbamyl transferase, carbamyl phosphate synthetase, pyruvate dehydrogenase, etc. were decreased $(6,22)$. In the ferret model exposed to aspirin, influenza, and AFD, arginase and other cytoplasmic enzyme activities were normal, whereas mitochondrial enzyme activities such as ornithine carbamyl transferase, carbamyl phosphate synthetase, and ornithine aminotransferase were decreased (11).

The hallmark of Reye's syndrome in both the human and the animal studies is selective involvement of mitochondrial metabolism with sparing of the cytosolic enzymes. We have shown that a combination of ibuprofen, influenza, and AFD results in both mitochondrial and cytoplasmic dysfunction as reflected in the reduced activities of hepatic arginase and ornithine carbamyl transferase. Additionally, the normal hepatic ornithine aminotransferase activity in these animals suggests that ibuprofen is not associated with panmitochondriopathy, which is observed in Reye's syndrome and in animals exposed to influenza, aspirin, and AFD (5). These results indicate that in infected and AFDtreated ferrets aspirin effect is more specific for mitochondrial injury, whereas ibuprofen results in generalized hepatopathy. The increased mortality in animals treated with ibuprofen, influenza, and AFD is thus associated with generalized hepatic damage rather than specific mitochondrial injury as observed with aspirin. It does not appear that ibuprofen is associated with Reye's syndrome-like changes in the ferret model.

\section{REFERENCES}

1. Reye RDK, Morgan G, Baral J 1963 Encephalopathy and fatty degeneration of the viscera: a disease entity in childhood. Lancet 2:249-252

2. Pranzarelli MR, De Vivo DC 1987 Pharmacology of Reye's syndrome. Clin Neuropharmacol 10:96-125

3. Kilpatric-Smith L, Hale DL, Douglas SD 1989 Progress in Reye's syndrome: epidemiology, biochemical mechanisms and animal models. Dig Dis Sci 7:135-146

4. Davis LE 1989 Reye's syndrome. Handb Clin Neurol 12:149-177

5. Deshmukh DR 1985 Animal models of Reye's syndrome. Rev Infec Dis 7:3140

6. Glasgow AM 1985 Overview of the metabolic abnormalities in Reye's syndrome. In: Pollack JD, Redshaw PL (eds) Reye's Syndrome IV. The National Reye's Syndrome Foundation, Bryan, OH, pp 142-152

7. Starko KM, Ray CG, Dominguez LB, Strombery WL, Woodal DF 1980 Reye's syndrome and salicylate use. Pediatrics 66:859-864

8. Hurwitz ES, Barret MJ, Bregman D, Gunn WJ, Schonberger LB, Fairweather WR, Drage JS, LaMontagne JR, Kaslow RA, Burlington B, Quinnan GV, Parker RA, Phillips K, Pinsky P, Dayton D, Dowdle WR 1987 Public health service study on Reye's syndrome and medication. JAMA 257:1905-1911

9. Pinsky PF, Hurwitz ES, Schonberger LB, Gunn WJ 1988 Reye's syndrome and aspirin: evidence for a dose-response effect. JAMA 260:657-661

10. Forsyth BW, Horwitz RI, Acampora D, Shapiro ED, Shapiro EG, Viscoli CM, Feinstein AR, Henner R, Holabird NB, Jones BA, Karabelas ADE, Kramer MS, Miclette M, Wells JA 1989 New epidemiologic evidence confirming that bias does not explain the aspirin/Reye's syndrome association. JAMA 261:2517-2524

11. Deshmukh DR, Maassab HF, Mason M 1982 Interactions of aspirin and other potential etiologic factors in an animal model of Reye's syndrome. Proc Natl Acad Sci USA 79:7557-7560

12. Morris JG, Rogers QR 1978 Arginine: an essential amino acid for cat. J Nutr 108:1944-1953

13. Mondzack A, Ehrlich GE, Sigmiller JE 1965 An enzymatic determination of ammonia in biological fluids. J Lab Clin Med 66:526-531

14. Kareman A 1955 A note on spectrophotometric assay of glutamic oxaloacetic transaminase in human blood serum. J Clin Invest 34:131-133

15. Cerriotti G 1974 Ornithine carbamyl transferase. In: Curtius HC, Roth M (eds) Clinical Biochemistry. Walter de Gruyter, Berlin, pp 1151-1156

16. Aravind MK, Miceli JN, Kauffman RE 1984 Determination of ibuprofen by high performance liquid chromatography. J Chromatogr 308:350-353

17. Tarrab R, Rodriguez J, Haitron C, Palacois R, Soberon G 1974 Molecular forms of rat liver arginase. Isolation and characterization. Eur J Biochem 49:457-468

18. Peraino C, Pitot HC 1963 Ornithine- $\delta$-transaminase. Biochim Biophys Acta 73:222-231

19. Bradford MM 1976 Rapid and sensitive method for quantification of microgram quantities of protein utilizing the principle of protein-dye binding. Anal Biochem 72:248-254

20. Linneman CC, Udea K, Hug G, Schaeffer A, Clark A, Schiff GM 1979 Salicylate intoxication and influenza in ferrets. Pediatr Res 13:44-47

21. Busson M 1986 Update on ibuprofen. J Int Med Res 14:53-62

22. DeVivo DC 1978 Reye's syndrome. A metabolic response to acute mitochondrial insult? Neurology 28:105-108 